PULIDO EE; BOFF P; DUARTE TS; BOFF MIC. 2014. Preparados homeopáticos en el crecimiento y en la producción de repollo cultivado en sistema orgánico. Horticultura Brasileira 32: 267-272. DOI - http://dx.doi.org/10.1590/S0102-05362014000300005

\title{
Preparados homeopáticos en el crecimiento y en la producción de repollo cultivado en sistema orgánico
}

\author{
Edwin E Pulido'; ${ }^{\text {; Pedro Boff }}{ }^{1,2}$; Tatiana S Duarte ${ }^{3}$; Mari IC Boff ${ }^{1}$ \\ ${ }^{1}$ UDESC-CAV, Depto. Prod. Vegetal, C. Postal 2090, 88520-000 Lages-SC; edwinpulido870@hotmail.com; a2micb@cav.udesc.br; \\ ${ }^{2}$ EPAGRI, Lab. de Homeopatia e Saúde Vegetal, C. Postal 181, 88506-080 Lages-SC; pboff@epagri.sc.gov.br; ${ }^{3}$ EPAGRI, EE Ituporanga, \\ C. Postal 121, 88400-000 Ituporanga-SC; tatianaduarte@epagri.sc.gov.br
}

\section{RESUMEN}

El repollo es consumido preferencialmente in natura y en muchos casos es producido de manera convencional, dejando trazas de residuos químicos, lo cual puede generar afecciones directas sobre la salud del consumidor y del agricultor. El objetivo del trabajo fue evaluar la influencia de preparados homeopáticos en altas diluciones en el crecimiento de plántulas y en la producción de repollo, en sistema orgánico. El trabajo fue compuesto por cinco experimentos para estudiar nueve tratamientos con cuatro repeticiones siendo la conducción de tres experimentos en invernadero, utilizando el diseño experimental de bloques completos al azar, y de dos experimentos en el campo utilizando el diseño experimental de bloques al azar. Los tratamientos estudiados fueron los preparados de Arnica montana, Silicea terra, Carbo vegetabilis y Sulphur en la $6 \mathrm{CH}$ y $30 \mathrm{CH}$ (orden de dilución centesimal hahnemanniana); y agua como testigo. Sulphur $6 \mathrm{CH}$ aumentó en dos de los tres experimentos la altura y la longitud de raíz en relación al testigo, en plántulas de repollo. Silicea terra $30 \mathrm{CH}$ y Sulphur $6 \mathrm{CH}$ aumentaron la producción y la materia seca de cabezas de repollo cultivadas en campo, así como, Arnica montana $6 \mathrm{CH}$ y $30 \mathrm{CH}$ también incrementaron la materia seca de cabezas. Se concluyó que Sulphur $6 \mathrm{CH}$ se destacó como promisorio para mejorar la calidad de las plántulas de repollo, así como en la producción y materia seca de cabezas de repollo en condiciones de campo siendo estas últimas características también mejoradas por Silicea terra $30 \mathrm{CH}$.

Palabras-clave: Brassica oleraceae var. capitata, homeopatía, prácticas agrícolas, sistema de cultivo.

\section{ABSTRACT}

Homeopathic preparations for growth and yield of cabbage in organic system

Cabbage is preferentially consumed in natura and in many cases is produced conventionally, leaving traces of chemical residues which can cause direct effects on the health of the consumer and the farmer. The aim was to evaluate the influence of high dilutions preparations on growth and production under organic system of cabbage evaluating seedlings and plants in field. The study was composed of five experiments with nine treatments and four replications. Three greenhouse and two field experiments were carried out in a randomized complete block design. The treatments were prepared of Arnica montana, Silicea terra, Carbo vegetabilis and Sulphur at $6 \mathrm{CH}$ and $30 \mathrm{CH}$ in high dilutions, water being used as control. Sulphur at $6 \mathrm{CH}$ increased the height and root length in comparison with a control, in two of the three experiments with cabbage seedlings. Silice terra at $30 \mathrm{CH}$ and Sulphur at $6 \mathrm{CH}$ increased the production and the dry mass of cabbage heads. Arnica montana at $6 \mathrm{CH}$ and $30 \mathrm{CH}$ also increased the dry mass. In conclusion, Sulphur at $6 \mathrm{CH}$ stands out as promising for improving the quality of cabbage seedlings as much as the production and dry mass of cabbage heads in field conditions, these last characteristics also being improved by Silicea terra at $30 \mathrm{CH}$.

Keywords: Brassica oleraceae var. capitata, homeopathy, agricultural practices, cropping system.

(Recebido para publicação em 3 de setembro de 2013; aceito em 6 de junho de 2014)

(Received on September 3, 2013; accepted on June 6, 2014)

$\mathrm{E}^{1}$ 1 repollo (Brassica oleraceae var. capitata) es la hortaliza más importante de las brásicas debido a la amplia distribución, facilidad de producción y alto consumo. Su cadena productiva presenta un alto impacto a nivel socioeconómico, debido a la generación de empleos en las diferentes etapas del cultivo hasta su poscosecha (Brackmann et al., 2003). El repollo es rico en vitaminas, principalmente en vitamina $\mathrm{C}$, además presenta alto contenido de sales minerales y aminoácidos esenciales como metionina y cisteína (Lédo et al., 2000).

El repollo es consumido, preferencialmente, in natura, lo cual exige que se adopten tecnologías limpias para su cultivo y producción, garantizando un producto libre de riesgos a la salud humana. El aumento de cultivos en sistemas orgánicos de producción es reflejo del consumo consciente que estimula la agricultura de bajo impacto y de prácticas saludables (Souza \& Alcântara, 2003).
La producción orgánica favorece y restablece el equilibrio de los agroecosistemas, el aumento de la biodiversidad, la preservación ambiental y el aprovechamiento eficiente de los recursos renovables, además del aporte en la calidad nutricional de los productos cuando son producidos en estos sistemas (Sarandón, 2008).

Técnicas saludables y que garanticen la alta calidad en la producción de repollo deben ser implementadas en las primeras etapas del cultivo, siendo 
la obtención de plántulas una de ellas (Guimarães et al., 2002). El sistema por trasplante, como la respectiva producción de plántulas, ofrece grandes ventajas en la optimización de los procesos, entre los cuales se destaca el mejor aprovechamiento de semillas, la adecuada planificación de la siembra, el mejor desarrollo radicular y el mejor manejo de las prácticas culturales en los primeros estados fenológicos como el deshierbe, el riego y el manejo fitosanitario (Jaramillo \& Diaz, 2006). Para la producción de cultivos en sistema orgánico, el manejo fitosanitario en base ecológica debe estar presente tanto en la obtención de plántulas como en la etapa de trasplante.

Preparados homeopáticos en altas diluciones han sido indicados como promisorios en el manejo fitosanitario de cultivos, siendo estos acordes a los sistemas orgánicos de producción, ya que cuando son aplicados a los cultivos generan un mínimo impacto ambiental. Otra de sus características es la fabricación a bajo costo, favoreciendo el acceso a estos preparados en altas diluciones por parte de las comunidades rurales, constituyéndose así en una tecnología de gran alcance social (Boff et al., 2008).

En el cultivo de hortalizas, el uso de preparados homeopáticos ha sido estudiado por varios autores. Rossi et al. (2006) encontraron mayor longitud de raíz y altura de plántulas de lechuga (Lactuca sativa) con la aplicación de Carbo vegetabilis con las dinamizaciones de $100 \mathrm{CH}$ y $200 \mathrm{CH}$ $(\mathrm{CH}=$ orden de dilución centesimal hahnemanniana). Modolon et al. (2012) concluyeron que el preparado de Arnica montana con la dinamización de $12 \mathrm{DH}$ mejoró la producción de frutos de tomate en campo. Bonato et al. (2009) identificaron que Sulphur en las dinamizaciones $6 \mathrm{CH}, 12 \mathrm{CH}, 24 \mathrm{CH}$ y $30 \mathrm{CH}$ aplicado en plantas de menta (Mentha arvensis) incrementó la altura de las plantas, el contenido de aceite esencial y las materia fresca y seca.

El objetivo del trabajo fue evaluar la influencia de preparados homeopáticos en altas diluciones en el crecimiento de plántulas en semillero y en la producción de repollo en campo, cultivado en sistema orgánico de producción.

\section{MATERIAL Y MÉTODOS}

Los estudios fueron realizados en la Estación Experimental de Lages de la EPAGRI (Empresa de Pesquisa Agropecuária e Extensão Rural de Santa Catarina) y en una finca de tradición agrícola localizada en la vereda de Pedras Brancas, Lages-SC, Brasil (2748'S, 50¹9'O, $931 \mathrm{~m}$ de altitude), en diferentes ciclos de cultivo de los años 2011 y 2012. La variedad utilizada para todos los experimentos fue el repollo híbrido Fuyutoyo.

Obtención de los preparados homeopáticos en altas diluciones - Los preparados homeopáticos en altas diluciones fueron obtenidos en el Laboratorio de Homeopatía y Salud Vegetal de la EPAGRI. Para la elaboración de los preparados homeopáticos en altas diluciones fue seguida la metodología descrita en la Farmacopea Homeopática Brasileña (1997). Las dinamizaciones fueron hechas a partir de las matrices en la dinamización de $5 \mathrm{CH}$, siendo utilizada una parte de la matriz en 99 partes de alcohol al 5\% y sucusionadas por 100 veces con auxilio del brazo mecánico (Autic $^{\circledR}$, Modelo Denise 10-50), para llegar a la dinamización de $6 \mathrm{CH}$ y así sucesivamente hasta alcanzar la dinamización deseada.

\section{Experimentos bajo invernadero}

- Los experimentos con plántulas de repollo fueron tres, realizados no invernadero, entre 30/09/2011 y $01 / 10 / 2012$, utilizando el diseño experimental de bloques completos al azar con cuatro repeticiones. Los tratamientos utilizados fueron los preparados homeopáticos en altas diluciones de $6 \mathrm{CH}$ y $30 \mathrm{CH}$ de Arnica montana, Silicea terra, Carbo vegetabilis y Sulphur y del agua como testigo. La parcela experimental fue compuesta por 64 plántulas de una bandeja de poliestireno expandido, con capacidad para 128 alveolos, constituyéndose la parcela útil por 20 plantas centrales. El sustrato orgánico utilizado para llenar los alveolos de las bandejas fue compuesto, en la proporción en volumen, por 3,0 partes de estiércol bovino; 1,0 de suelo; 1,0 de vermiculita y 0,2 de fosfato natural y densidad media de los componentes, en $\mathrm{kg} \mathrm{L}^{-1}$, de 0,$7 ; 1,0 ; 0,08$ y de 2,8, respectivamente. En el momento de la mezcla todos los materiales estaban secos para la correcta elaboración del sustrato.

En la siembra, fueron depositadas dos semillas por alveolo, a $0,5 \mathrm{~cm}$ de profundidad. Una semana después de la emergencia fue realizado el raleo, dejando una plántula por alveolo. El riego de las plántulas fue hecho diariamente por microaspersión durante tres minutos y repetido en los momentos en los cuales las plantas presentaban déficit hídrico, de acuerdo a una inspección visual hecha en el sustrato.

Las aplicaciones sobre las plántulas de los preparados homeopáticos en altas diluciones y del agua fueron siete, realizadas a cada cuatro días, hasta su trasplante, con aspersor manual Vonder ${ }^{\mathbb{R}}$ con capacidad para 1,5 L. Las bandejas fueron aisladas unas de las otras en el momento de la pulverización. Cada preparado homeopático fue utilizado en la dosis de $10 \mathrm{~mL}$ para cada litro de agua y homogeneizado con diez agitaciones. Se utilizó la técnica de double-blind para la obtención de los preparados y la pulverización de los mismos, donde ni el evaluador ni el aplicador conocían la naturaleza de cada tratamiento.

Las evaluaciones fueron realizadas cuando las plántulas alcanzaron más del $70 \%$ del estado fenológico $\mathrm{V}_{0}$, caracterizado por plántulas con tres a cuatro hojas verdaderas y altura de 10 a $12 \mathrm{~cm}$ (Jaramillo \& Diaz, 2006). Las plántulas fueron retiradas de las bandejas para analizar las características de altura de la planta, longitud de la mayor raíz, diámetro del tallo y materias frescas y secas de la parte aérea y de la radicular. Para la evaluación de la materia seca los materiales húmedos fueron llevados a la estufa de circulación de aire forzado, a $65^{\circ} \mathrm{C}$, hasta tener un peso constante.

Experimentos en el campo - Los experimentos en el campo fueron dos y realizados durante los periodos de 13/12/2011 al 17/04/2012 (área 1) y de 14/05/2012 al 17/09/2012 (área 2) en 
la ciudad de Lages-SC, Brasil. El clima predominante en la región es templado húmedo, con temperatura media del aire en el mes más caluroso de $22^{\circ} \mathrm{C}$ y en los meses de invierno entre 6 a $8^{\circ} \mathrm{C}$. La precipitación pluvial total anual varia de 1.360 a $1.600 \mathrm{~mm}$ y los suelos son clasificados como "Cambissolo Húmico Álico” (EPAGRI/CIRAM, 2002).

Los análisis químicos de los suelos donde fueron realizados los experimentos presentaron las siguientes características: Área $1=38 \%$ de arcilla $\left(\mathrm{m} \mathrm{v}^{-1}\right) ; 5,3$ de pH-agua $(1: 1) ; 3,0 \%$ de M.O. $\left(\mathrm{m} \mathrm{v}^{-1}\right) ; 5,9$ cmolc $\mathrm{L}^{-1}$ de $\mathrm{Ca} ; 2,0$ cmolc $\mathrm{L}^{-1}$ de $\mathrm{Mg}$; 0,3 cmolc $\mathrm{L}^{-1}$ de $\mathrm{Al}$; $18,5 \mathrm{mg} \mathrm{L}^{-1}$ de P y $213 \mathrm{mg} \mathrm{L}^{-1}$ de $\mathrm{K}$. Área 2: $32 \%$ de arcilla $\left(\mathrm{m} \mathrm{v}^{-1}\right) ; 5,5 \mathrm{de}$ pH-agua (1:1); 3,7\% M.O. $\left(\mathrm{m} \mathrm{v}^{-1}\right) ; 6,6$

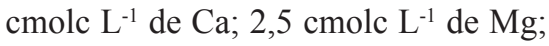
0,0 cmolc $\mathrm{L}^{-1}$ de Al; 32,4 $\mathrm{mg} \mathrm{L}^{-1}$ de $\mathrm{P}$ y $171 \mathrm{mg} \mathrm{L}^{-1}$ de $\mathrm{K}$.

Los tratamientos utilizados fueron los preparados homeopáticos $6 \mathrm{CH}$ y $30 \mathrm{CH}$ de Arnica montana, Silicea terra, Carbo vegetabilis e Sulphur y del agua como testigo. Los dos experimentos fueron compuestos por nueve tratamientos arreglados en el diseño experimental de bloques completos al azar, con cuatro repeticiones. Cada parcela fue compuesta por 10 plantas distribuidas en doble hilera.

Las plántulas fueron producidas en bandejas de poliestireno expandido de 128 alveolos. El sustrato orgánico utilizado para llenar los alveolos de las bandejas fue compuesto, en la proporción en volumen, por 3,0 partes de estiércol bovino, 1,0 de suelo, 1,0 de vermiculita y 0,2 de fosfato natural y densidad media de los componentes, en $\mathrm{kg} \mathrm{L}^{-1}$, de 0,$7 ; 1,0 ; 0,08$ y de 2,8, respectivamente. En el momento de la mezcla todos los materiales estaban secos para la correcta elaboración del sustrato.

La preparación del suelo fue realizada con arado y gradeo, en un área que en su ciclo anterior estaba sembrado abono verde de avena y arveja. Para la fertilización fueron aplicados $2,5 \mathrm{~L} \mathrm{~m}^{-2}$ de estiércol bovino compuesto por: $48 \%$ de $\mathrm{C} ; 2,3 \%$ de $\mathrm{N}$ en la materia seca; 4,1 $\mathrm{kg} \mathrm{m}{ }^{-2}$ de $\mathrm{P}_{2} \mathrm{O}_{5} ; 3,8 \mathrm{~kg} \mathrm{~m}^{-2}$ de $\mathrm{K}_{2} \mathrm{O}$ y 3,0 $\mathrm{kg} \mathrm{m}^{-2} \mathrm{de} \mathrm{Ca}$, además de adicionarse 0,15 $\mathrm{kg} \mathrm{m}^{-2}$ de fosfato natural.
El trasplante fue realizado cuando las plántulas de repollo se encontraban en el estado fenológico $\mathrm{V}_{0}$, conforme Jaramillo \& Diaz (2006), caracterizado por plántulas con tres a cuatro hojas verdaderas y altura de 10 a $12 \mathrm{~cm}$. Momentos antes del transplante, las plántulas fueron lavadas con agua y desinfectadas con hipoclorito de sodio al 2,5\% de cloro activo, en la dosis de 5 $\mathrm{mL} \cdot \mathrm{L}^{-1}$ de agua. La siembra del repollo consistió en dejar una planta por hoyo $\mathrm{y}$ en distancias de $0,4 \mathrm{~m}$ entre plantas y $0,6 \mathrm{~m}$ entre hileras, correspondiendo a la densidad de 41.500 plantas por hectárea.

Los tratamientos fueron aplicados cada quince días, a partir de los siete días después del trasplante hasta el momento de cosecha, totalizando cuatro aplicaciones. Las aplicaciones fueron realizadas sobre las plantas con bomba de espalda Guarany ${ }^{\circledR}$ con capacidad para 5 L. Cada preparado homeopático en alta dilución fue utilizado en la dosis de $10 \mathrm{~mL}$ para cada litro de agua y homogeneizado con diez agitaciones. Se utilizó la técnica de double-blind para la aplicación de los tratamientos, donde ni el evaluador ni el aplicador conocían la naturaleza de cada tratamiento, siendo estos identificados solamente después de los análisis estadísticos.

El riego fue conforme las necesidades de las plantas cultivadas y el manejo de las hierbas fue a través de deshierbas manuales.

En el momento de la cosecha, correspondiente al estado fenológico $\mathrm{R}_{2}$ (la forma de la cabeza es de aproximadamente 15,25 - 30,48 cm de diámetro, no hay producción de hojas nuevas después que la cabeza llega a su tamaño máximo), fueron evaluados el índice de formato - que es la relación entre el diámetro longitudinal y transversal de la cabeza del repollo y la relación $\mathrm{C} / \mathrm{D}$ - que expresa la longitud del corazón en relación a la profundidad de la cabeza. Después de la remoción de las hojas externas de las plantas del repollo, se determinó la materia fresca de las cabezas. Para la materia seca fueron seleccionadas tres cabezas de repollo por parcela y acondicionadas en bolsas de papel Kraft y llevadas a la estufa de circulación de aire forzado a $65^{\circ} \mathrm{C}$, hasta tener un peso constante.
Los datos fueron almacenados, procesados y analizados por medio del Sistema de Análisis Estadístico para Windows (WinStat, v. 1.0) (Machado \& Conceição, 2009), donde fue aplicado análisis de varianza ANOVA $\mathrm{y}$ las medias, cuando $\mathrm{F}, \mathrm{p} \leq 0,05$, fueron comparadas por el test de Tukey, a 5\% de probabilidad. Se realizó análisis de correlación de Pearson por el test T, a $5 \%$ de probabilidad.

\section{RESULTADOS Y DISCUSIÓN}

En el experimento 1, los preparados de Sulphur 6CH, Silicea terra $30 \mathrm{CH}$ e Arnica montana $6 \mathrm{CH}$ indujeron aumentos de 0,044; 0,036 e 0,031 g planta $^{-1}$, respectivamente, de materia seca de parte aérea de las plántulas de repollo en relación a la materia seca de las plántulas tratadas con Carbo vegetabilis, que presentó 0,098 g planta $^{-1}$ como el menor valor (Tabla 1). En los experimentos 2 y 3 no se encontraron diferencias significativas entre los tratamientos, referentes a la materia seca de las plántulas de repollo. En estudios realizados por Bonato \& Silva (2003), también se demostró la influencia del preparado Sulphur en la $5 \mathrm{CH}$ y $12 \mathrm{CH}$, promoviendo el aumento de materia seca de la parte aérea en plantas de rábano (Raphanus sativus).

En relación a la materia seca del sistema radicular las respuestas de las plantas a los tratamientos aplicados fueron diferentes en cada experimento (Tabla 1). En dos de los tres experimentos los valores del tratamiento con Arnica montana $6 \mathrm{CH}$ fueron los mayores y se diferenciaron en 0,015 y 0,019 g planta $^{-1}$ en relación a los valores obtenidos por las plantas del tratamiento testigo, que alcanzaron los menores valores. Estas diferencias pueden ser explicadas por Bonato et al. (2009), donde el aumento del metabolismo en las plantas tratadas con preparados en altas diluciones es debido probablemente al incremento de la producción de carbono para el crecimiento.

El análisis de correlación de Pearson presentó valores significativos, entre materia fresca y seca de la parte aérea en las plántulas de repollo en el 
Tabla 1. Materia seca de las partes aérea y radicular de plántulas de repollo híbrido Fuyutoyo tratadas con preparados homeopáticos en altas diluciones (shoo777t dry mass and root dry mass of seedlings of cabbage Fuyutoyo hybrid treated with homeopathic preparations in high dilution). Lages, UDESC, 2011/2012.

\begin{tabular}{|c|c|c|c|c|c|c|}
\hline \multirow[t]{2}{*}{ Preparados } & \multicolumn{3}{|c|}{$\begin{array}{c}\text { Materia seca de la parte aérea } \\
\text { (g/planta) }\end{array}$} & \multicolumn{3}{|c|}{$\begin{array}{c}\text { Materia seca de la parte radicular } \\
\text { (g/planta) }\end{array}$} \\
\hline & Exp 1 & $\operatorname{Exp} 2$ & $\operatorname{Exp} 3$ & $\operatorname{Exp} 1$ & $\operatorname{Exp} 2$ & $\operatorname{Exp} 3$ \\
\hline Arnica montana $6 \mathrm{CH}$ & 0,129 abc & 0,142 & 0,132 & $0,045 \mathrm{ab}$ & 0,044 a & 0,049 a \\
\hline Arnica montana $30 \mathrm{CH}$ & $0,104 \mathrm{~cd}$ & 0,116 & 0,109 & $0,033 \mathrm{~b}$ & $0,032 \mathrm{ab}$ & $0,032 \mathrm{bc}$ \\
\hline Carbo vegetabilis $6 \mathrm{CH}$ & $0,098 \mathrm{~d}$ & 0,111 & 0,101 & $0,028 \mathrm{~b}$ & $0,030 \mathrm{~b}$ & $0,031 \mathrm{bc}$ \\
\hline Carbo vegetabilis $30 \mathrm{CH}$ & $0,120 \mathrm{abcd}$ & 0,115 & 0,110 & $0,038 \mathrm{~b}$ & $0,031 \mathrm{ab}$ & $0,035 \mathrm{abc}$ \\
\hline Silicea terra $6 \mathrm{CH}$ & $0,109 \mathrm{bcd}$ & 0,118 & 0,108 & $0,040 \mathrm{~b}$ & $0,033 \mathrm{ab}$ & $0,033 \mathrm{bc}$ \\
\hline Silicea terra $30 \mathrm{CH}$ & $0,134 \mathrm{ab}$ & 0,128 & 0,123 & $0,043 \mathrm{ab}$ & $0,039 \mathrm{ab}$ & $0,047 \mathrm{ab}$ \\
\hline Sulphur 6CH & $0,142 \mathrm{a}$ & 0,140 & 0,125 & 0,059 a & $0,036 \mathrm{ab}$ & $0,038 \mathrm{abc}$ \\
\hline Sulphur 30CH & $0,127 \mathrm{abcd}$ & 0,130 & 0,123 & 0,058 a & $0,038 \mathrm{ab}$ & $0,040 \mathrm{abc}$ \\
\hline Agua & $0,110 \mathrm{bcd}$ & 0,108 & 0,106 & $0,033 \mathrm{~b}$ & $0,029 \mathrm{~b}$ & $0,030 \mathrm{c}$ \\
\hline CV (\%) & 10,44 & 12,00 & 13,95 & 17,51 & 15,76 & 17,72 \\
\hline
\end{tabular}

Valores promedio seguidos de la misma letra, en las columnas, no difieren entre sí, por el test de Tukey, al 5\% de probabilidad (means followed by the same letter in the column did not differ from each other by Tukey test 5\%). Valores no acompañados por letras significan que no fueron diferentes por el test $\mathrm{F}$ (values not accompanied by letters mean that were not different by $\mathrm{F}$ test). Exp $1=\operatorname{experimento~} 1$. Exp 2 = experimento 2. Exp 3 = experimento 3 .

Tabla 2. Altura, longitud de la mayor raíz y diámetro del tallo de plántulas de repollo híbrido Fuyutoyo (plant height, root length and stem diameter of cabbage hybrid Fuyutoyo seedlings). Lages, UDESC, 2011/2012.

\begin{tabular}{|c|c|c|c|c|c|c|c|c|c|}
\hline \multirow{2}{*}{ Preparados } & \multicolumn{3}{|c|}{ Altura (cm) } & \multicolumn{3}{|c|}{ Longitud de la mayor raíz (cm) } & \multicolumn{3}{|c|}{ Diámetro del tallo (cm) } \\
\hline & $\operatorname{Exp} 1$ & $\operatorname{Exp} 2$ & Exp 3 & $\operatorname{Exp} 1$ & $\operatorname{Exp} 2$ & $\operatorname{Exp} 3$ & $\operatorname{Exp} 1$ & $\operatorname{Exp} 2$ & $\operatorname{Exp} 3$ \\
\hline Arnica montana $6 \mathrm{CH}$ & 12,0 & $12,5 \mathrm{ab}$ & $11,9 \mathrm{ab}$ & $8,9 \mathrm{bc}$ & $8,8 \mathrm{ab}$ & $9,4 \mathrm{ab}$ & $1,1 \mathrm{ab}$ & 1,2 & 1,2 \\
\hline Arnica montana $30 \mathrm{CH}$ & 11,0 & $12,3 \mathrm{ab}$ & $12,4 \mathrm{ab}$ & $8,5 \mathrm{bc}$ & $8,4 \mathrm{ab}$ & $8,9 \mathrm{ab}$ & $1,0 \mathrm{ab}$ & 1,2 & 1,1 \\
\hline Carbo vegetabilis $6 \mathrm{CH}$ & 12,0 & $12,1 \mathrm{ab}$ & $11,8 \mathrm{ab}$ & $7,4 \mathrm{c}$ & $8,3 \mathrm{ab}$ & $9,1 \mathrm{ab}$ & $1,2 \mathrm{ab}$ & 1,1 & 1,1 \\
\hline Carbo vegetabilis $30 \mathrm{CH}$ & 12,0 & $12,3 \mathrm{ab}$ & $12,0 \mathrm{ab}$ & $9,1 \mathrm{abc}$ & 9,8 a & $9,3 \mathrm{ab}$ & $1,2 \mathrm{ab}$ & 1,1 & 1,1 \\
\hline Silicea terra $6 \mathrm{CH}$ & 12,1 & $11,4 \mathrm{ab}$ & $11,4 \mathrm{ab}$ & $8,5 \mathrm{bc}$ & $8,1 \mathrm{ab}$ & $8,3 \mathrm{ab}$ & $0,9 \mathrm{~b}$ & 1,2 & 1,1 \\
\hline Silicea terra $30 \mathrm{CH}$ & 12,5 & $12,3 \mathrm{ab}$ & $12,3 \mathrm{ab}$ & $8,9 \mathrm{bc}$ & $8,7 \mathrm{ab}$ & 9,9 a & $1,2 \mathrm{ab}$ & 1,2 & 1,2 \\
\hline Sulphur $6 \mathrm{CH}$ & 13,2 & $12,8 \mathrm{a}$ & 12,8 a & $10,4 \mathrm{ab}$ & $10,0 \mathrm{a}$ & $9,2 \mathrm{ab}$ & $1,3 \mathrm{a}$ & 1,1 & 1,1 \\
\hline Sulphur $30 \mathrm{CH}$ & 12,9 & $13,1 \mathrm{a}$ & 13,6 a & $11,2 \mathrm{a}$ & $8,4 \mathrm{ab}$ & $9,6 \mathrm{ab}$ & $1,1 \mathrm{ab}$ & 1,1 & 1,1 \\
\hline Agua & 11,3 & $10,4 \mathrm{~b}$ & $10,1 \mathrm{~b}$ & $7,2 \mathrm{c}$ & $7,0 \mathrm{~b}$ & $7,6 \mathrm{~b}$ & $0,9 \mathrm{~b}$ & 1,1 & 1,0 \\
\hline CV (\%) & 12,7 & 11,7 & 13,3 & 10,2 & 11,8 & 10,1 & 9,3 & 10,3 & 9,4 \\
\hline
\end{tabular}

Valores promedio seguidos de la misma letra, en las columnas, no difieren entre sí, por el test de Tukey, al 5\% de probabilidad (means followed by the same letter in the column did not differ from each other by Tukey test $5 \%$ ). Valores no acompañados por letras significan que no fueron diferentes por el test $\mathrm{F}$ (values not accompanied by letters mean that they were not different by $\mathrm{F}$ test). Exp $1=$ experimento 1. $\operatorname{Exp} 2=$ experimento 2. Exp $3=$ experimento 3 .

experimento uno $(\mathrm{r}=0,61 ; \mathrm{p}>0,05, \mathrm{n}=$ 36), experimento dos $(r=0,61 ; p>0,05$, $\mathrm{n}=36)$ y experimento tres $(\mathrm{r}=0,45$; $\mathrm{p}>0,05, \mathrm{n}=36)$. Al presentarse una correlación significativa entre la materia fresca y seca, los análisis estadísticos fueron hechos con los valores de materia seca de las plántulas de repollo (Tabla $1)$.

La correlación entre materia seca de la parte aérea y radicular también fue significativa para el experimento uno $(\mathrm{r}=$
0,$65 ; p>0,05, n=36$ ), experimento dos $(\mathrm{r}=0,80 ; \mathrm{p}>0,05, \mathrm{n}=36)$ y experimento tres $(r=0,46 ; p>0,05, n=36)$ (Tabla 1$)$. Estas correlaciones positivas indican que el aumento de materia seca de la parte radicular favorece el aumento de la materia seca de la parte aérea, es decir, un óptimo crecimiento de raíces como resultado de la producción de plántulas de mejor calidad, así como un desarrollo adecuado de la parte aérea.

Las alturas de las plántulas de repollo de los tratamientos Sulphur $30 \mathrm{CH}$ y $6 \mathrm{CH}$ fueron las mayores en el segundo experimento y se diferenciaron en 2,70 y $2,40 \mathrm{~cm}$, respectivamente, $y$ en el tercer experimento en 3,50 y $2,70 \mathrm{~cm}$, en relación al tratamiento testigo, siendo este el de menor altura de la plántula de 10,4 y $10,1 \mathrm{~cm}$ en dichos experimentos (Tabla 2). Estos preparados de Sulphur en las dinamizaciones $30 \mathrm{CH}$ y $6 \mathrm{CH}$ proporcionaron mayor altura de las plántulas de repollo en comparación a 
Tabla 3. Producción de cabezas, materia seca de cabezas, índice de formato y relación C/D de plantas de repollo híbrido Fuyutoyo tratadas con preparados homeopáticos en altas diluciones (production, dry mass, shape index and rate C/D of cabbage Fuyutoyo hybrid plants treated with homeopathic preparations in high dilution). Lages, UDESC, 2011/2012.

\begin{tabular}{|c|c|c|c|c|c|c|c|c|}
\hline \multirow{2}{*}{ Preparados } & \multicolumn{2}{|c|}{ Producción (t ha $\left.{ }^{-1}\right)$} & \multicolumn{2}{|c|}{ Materia seca de cabezas (g) } & \multicolumn{2}{|c|}{ Índice de formato $^{1}$} & \multicolumn{2}{|c|}{$\mathbf{C} / \mathbf{D}^{2}$} \\
\hline & Área 1 & Área 2 & Área 1 & Área 2 & Área 1 & Área 2 & Área 1 & Área 2 \\
\hline Arnica montana $6 \mathrm{CH}$ & $42,38 \mathrm{ab}$ & $46,03 \mathrm{ab}$ & 205,26 a & $231,03 \mathrm{ab}$ & 0,78 & 0,92 & 0,50 & 0,46 \\
\hline Arnica montana $30 \mathrm{CH}$ & $30,44 \mathrm{c}$ & $37,19 \mathrm{~b}$ & $147,35 \mathrm{~b}$ & $226,00 \mathrm{abc}$ & 0,75 & 0,94 & 0,45 & 0,45 \\
\hline Carbo vegetabilis $6 \mathrm{CH}$ & $33,45 \mathrm{bc}$ & $36,88 \mathrm{~b}$ & $154,53 \mathrm{~b}$ & $160,77 \mathrm{~cd}$ & 0,72 & 0,97 & 0,47 & 0,42 \\
\hline Carbo vegetabilis $30 \mathrm{CH}$ & $38,33 \mathrm{abc}$ & $41,15 \mathrm{ab}$ & $143,73 \mathrm{~b}$ & $158,33 \mathrm{~d}$ & 0,79 & 0,91 & 0,48 & 0,45 \\
\hline Silicea terra $6 \mathrm{CH}$ & $38,95 \mathrm{abc}$ & $34,17 \mathrm{~b}$ & $165,47 \mathrm{ab}$ & $167,45 \mathrm{bcd}$ & 0,80 & 0,97 & 0,46 & 0,40 \\
\hline Silicea terra $30 \mathrm{CH}$ & 44,39 a & $42,37 \mathrm{ab}$ & $176,43 \mathrm{ab}$ & $252,20 \mathrm{a}$ & 0,75 & 0,99 & 0,51 & 0,42 \\
\hline Sulphur $6 \mathrm{CH}$ & $37,70 \mathrm{abc}$ & 50,42 a & $150,52 \mathrm{~b}$ & $232,73 \mathrm{ab}$ & 0,75 & 0,95 & 0,47 & 0,46 \\
\hline Sulphur $30 \mathrm{CH}$ & $38,57 \mathrm{abc}$ & $39,20 \mathrm{ab}$ & $163,81 \mathrm{ab}$ & $167,00 \mathrm{bcd}$ & 0,90 & 0,97 & 0,43 & 0,42 \\
\hline Agua & $32,34 \mathrm{bc}$ & $35,65 \mathrm{~b}$ & $155,78 \mathrm{~b}$ & $126,29 \mathrm{~d}$ & 0,79 & 0,96 & 0,49 & 0,40 \\
\hline CV (\%) & 11,51 & 13,53 & 11,15 & 14,48 & 13,13 & 10,59 & 7,20 & 8,05 \\
\hline
\end{tabular}

Valores promedio seguidos de la misma letra, en las columnas, no difieren entre sí, por el test de Tukey, al 5\% de probabilidad (means followed by the same letter in the column did not differ from each other by Tukey test 5\%). Valores no acompañados por letras significan que no fueron diferentes por el test F (values not accompanied by letters mean that there were no difference by F test). Área 1= Estación Experimental de Lages. Área 2= Vereda Pedras Brancas; 'Índice de formato = relación entre los diámetros longitudinal y transversal de la cabeza (shape index $=$ rate of the longitudinal and transverse diameters of the cabbage head). ${ }^{2} \mathrm{C} / \mathrm{D}=$ relación entre la longitud del corazón y el diámetro de la cabeza $(\mathrm{C} / \mathrm{D}=$ rate of the length of the heart and longitudinal diameter).

las descritas por Magro et al. (2011), que fueron de $10,52 \mathrm{~cm}$ y producidas convencionalmente. Alturas mayores en las plántulas dan como resultado una elevada tasa de sobrevivencia y de crecimiento inicial, favoreciendo el trasplante en campo. Resultados semejantes fueron presentados por Bonato et al. (2009) al utilizar el preparado de Sulphur 6CH en el incremento de algunas características de las plantas de menta (Mentha arvensis) como la altura y el contenido de aceite esencial.

Para la longitud de la mayor raíz hubo respuestas variables en cada experimento, siendo que las plantas del tratamiento testigo siempre presentaron los menores valores de 7,2; 7,0 y 7,6 $\mathrm{cm}$, respectivamente (Tabla 2). Hubo diferencias significativas del tratamiento testigo con los valores de algunos tratamientos como del Sulphur 30CH y $6 \mathrm{CH}$ de 4,0 y $3,2 \mathrm{~cm}$ en el primer experimento; de Sulphur $6 \mathrm{CH}$ y Carbo vegetabilis $30 \mathrm{CH}$ de 3,0 y $2,8 \mathrm{~cm}$ en el segundo experimento; y de Silicea terra $30 \mathrm{CH}$ de $2,3 \mathrm{~cm}$ en el tercer experimento (Tabla 2). Ninguno de los preparados homeopáticos en altas diluciones inhibió la longitud de la raíz en las plántulas de repollo, así estos no fueran significativamente diferentes con el tratamiento testigo. El adecuado crecimiento del sistema radicular es uno de los factores más importantes en la producción de plántulas de las brásicas. Sistemas radiculares profundos aumentan el área de contacto entre las raíces y el suelo, ofreciendo mayor resistencia al estrés hídrico. En el momento del trasplante, las plántulas con sistemas radiculares cortos son más vulnerables, debido a la escasa disponibilidad de agua en la capa superficial y a los factores abióticos como precipitación y vientos (Jaramillo \& Diaz, 2006).

Para el diámetro del tallo, apenas Sulphur en la $6 \mathrm{CH}$ presentó diferencias significativas en uno de los experimentos de $0,4 \mathrm{~cm}$ frente al testigo, de $0,9 \mathrm{~cm}$ (Tabla 2). De acuerdo con Bonato \& Silva (2003), es frecuente que en la ciencia homeopática, el mismo medicamento cause efectos diferentes y ocurra un acción estimulante o inhibitoria en las características consideradas.

En el campo, el preparado de Silicea terra $30 \mathrm{CH}$, en el área 1, promovió el aumento de la producción de cabezas de repollo híbrido Fuyutoyo en 13,95 t ha ${ }^{-1}$, en comparación con Arnica montana $30 \mathrm{CH}$ que fue el tratamiento con menor producción de $30,44 \mathrm{t} \mathrm{ha}^{-1}$ (Tabla 3). Resultado observado también por Almeida (2002) cuando verificó el incremento de la producción de albahaca (Ocimum basilicum) con el uso de Silicea terra $30 \mathrm{CH}$, aumentando en $40 \%$ la materia fresca de las inflorescencias. Sin embargo, este resultado no fue reflejado en el área 2, donde se obtuvo los mejores resultados de producción con Sulphur en la 6CH y se diferenciaron en 16,25 t ha $^{-1}$, en comparación con Silicea terra $6 \mathrm{CH}$ que fue el tratamiento con menor producción de 34,17 tha ${ }^{-1}$ (Tabla 3). En sistema convencional, la mayor producción obtenida con el híbrido Fuyutoyo por Carvalho \& Ikuta (2003) fue de $48,13 \mathrm{t} \mathrm{ha}^{-1}$, el cual se aproxima a los valores alcanzados en nuestro trabajo realizado en sistema orgánico. Por otro lado, cuando se utilizó Sulphur en la $6 \mathrm{CH}$, la producción de cabezas de repollo del híbrido Fuyutoyo fue superior en relación al tratamiento testigo (Tabla 3).

En relación a la materia seca de cabezas de repollo, el preparado de Arnica montana $6 \mathrm{CH}$ promovió el aumento en 49,48 $\mathrm{g}_{\text {planta }}{ }^{-1}$ para el área $1 \mathrm{y}$ en $104,74 \mathrm{~g}_{\text {planta }}{ }^{-1}$ para el área 2, frente al tratamiento testigo, de 155,78 g planta $^{-1}$ y 126,29 g planta $^{-1}$, respectivamente (Tabla 3). Resultados semejantes con el uso de Arnica montana en la $3 \mathrm{CH}, 6 \mathrm{CH}$ y 
$12 \mathrm{CH}$ fueron obtenidos por Bonfim et al. (2008), en el aumento de la materia seca de la parte aérea en romero (Rosmarinus officinalis) y en salve real (Lippia alba). Igualmente, Grisa et al. (2007), en estudios con plantas de lechuga tratadas con Arnica montana en las dinamizaciones $6 \mathrm{CH}$ y $12 \mathrm{CH}$ observaron aumento de materia seca de la parte aérea.

Para las características del índice de formato y la relación de longitud del corazón y diámetro de la cabeza (C/D), no fueron observadas diferencias significativas entre los tratamientos, tanto en los experimentos como en las diferentes áreas (Tabla 3). El repollo del híbrido Fuyutoyo presentó cabezas levemente achatadas en las dos áreas, siendo que la media general obtenida del índice de formato entre los tratamientos fue de 0,78 en el área uno y de 0,95 en el área dos. Sin embargo, la relación $\mathrm{C} / \mathrm{D}$ fue de 0,49 en el área uno y 0,43 en el área dos (Tabla 3).

Este resultado puede ser atribuido a que la carga genética que codifica la forma de la cabeza no fue influenciada por el medio ambiente o por los preparados homeopáticos en altas diluciones. Por su parte, Lédo et al. (2000) encontraron en repollo cultivado con manejo convencional, el índice de formato de 0,83 para el híbrido Fuyutoyo, siendo las cabezas caracterizadas como levemente achatadas, y la relación C/D de 0,57. Según Silva Júnior et al. (1988), valores elevados del índice de formato indican el grado de achatamiento de la cabeza, siendo los índices próximos a 1,0 los de mayor preferencia en el mercado de repollo. Por otro lado, valores elevados de la relación $\mathrm{C} / \mathrm{D}$ comprometen la calidad de las cabezas de repollo, pudiendo en ocasiones favorecer el rompimiento de esta $y$ afectar la vida útil del producto después de la madurez comercial.

En conclusión, preparados homeopáticos en altas diluciones influencian el crecimiento de plantas de repollo, en sistema orgánico, desde sus primeros estados fenológicos $\mathrm{V}_{0}$ (etapa de plántula) hasta $\mathrm{R}_{2}$ (etapa de la formación de cabezas). Sulphur $6 \mathrm{CH}$ mejoró la calidad de las plántulas de repollo, además que en condiciones de campo su respuesta fue significativa en la producción y materia seca de cabezas de repollo, siendo estas últimas características también mejoradas por el preparado de Silicea terra $30 \mathrm{CH}$.

\section{AGRADECIMIENTOS}

Al MCT/CNPq/MEC/CAPES/CT, HIDRO/FAPS/EMBRAPA a través del proyecto Repensa - 22/2010 por el apoyo financiero para el desarrollo de la investigación. A la FAPESC a través del proyecto Rede Guarani Serra Geral, conv. FAPEU/FAPESC 16261-10/2 y al proyecto Agrarias por su apoyo. El primer autor agradece a la CAPES por la adjudicación de la beca para estudios de maestría.

\section{REFERENCIAS}

ALMEIDA MAZ. 2002. Resposta do manjericão (Ocimum basilicum) à aplicação de preparações homeopáticas. Viçosa: UFV. $101 \mathrm{p}$ (Disertación de maestría).

BOFF P; GIESELA; MADRUGA E; SOUZA LT; BOFF MIC; GONÇALVES PAS; ZARDO VF. 2008. Agropecuária saudável: da prevenção de doenças, pragas e parasitas à terapêutica não residual. Lages: Epagri - UDESC. 80p.

BONATO CM; PROENÇA GT; REIS B. 2009. Homeopathic drugs Arsenicum album and Sulphur affect the growth and essential oil content in mint (Mentha arvensis). Acta Scientiarum 31: 101-105.

BONATO CM; SILVA EP. 2003. Effect of the homeopathic solution Sulphur on the growth and productivity of radish. Acta scientiaru 25: 259-263.

BONFIM FPG; MARTINS ER; DORES RG; BARBOSA CKR; CASALI VWD; HONÓRIO ICG. 2008. Use of homeopathic Arnica montana for the issuance of roots of Rosmarinus officinalis and Lippia alba. International Journal of High Dilutio 7: 113-117.

BRACKMANN A; TREVISAN JN; MARTINS GAK; FREITAS ST; MELLO AM. 2003. Etileno, 1-metilciclopropeno e qualidade de repolho cv. Wakaba armazenado em ambiente refrigerado. Revista Brasileira de Agrociência 9: 403-405.

CARVALHO RIN; IKUTA ARY. 2003.
Competição entre cultivar e híbridos de repolho no município de Piraquara, PR. Revista Acadêmica: Ciências Agrárias e Ambientais 1: 33-36.

EPAGRI/CIRAM，2002. Zoneamento agroecológico e socioeconômico do Estado de Santa Catarina. Disponible en http:// www.ciram.epagri.sc.gov.br/ciram_arquivos. Consultado en 12 de diciembre de 2012.

FARMACOPÉIA HOMEOPÁ TICA BRASILEIRA. 1997. São Paulo: Atheneu. 118p.

GRISA S; TOLEDO MV; OLIVEIRA LC; HOLZ L; MARINE D. 2007. Crescimento e produtividade de alface sob diferentes diluições do medicamento homeopático Arnica montana. Revista Brasileira de Agroecologia 2: 1050-1053.

GUIMARÃES VF; ECHER MM; MINAMI K. 2002. Métodos de produção de mudas, distribuição de matéria seca e produtividade de plântulas de beterraba. Horticultura Brasileira 20: 505-509.

JARAMILLO NJE; DIAZ DCA. 2006. Generalidades del cultivo. In: JARAMILLO NJE; DIAZ DCA. El cultivo de las cruciferas. Rionegro: CORPOICA, Centro de Investigación La Selva. Manual Técnico 20: 9-55.

LÉDO FJS; SOUZA JA; SILVA MR. 2000. Avaliação de cultivares e híbridos de repolho no Estado do Acre. Horticultura Brasileira 18: $138-140$.

MACHADO AM; CONCEIÇÃO AR. 2009. Sistema de análise estatística para Windows - WinStat. Versão 1.0. Pelotas: UFPel/Nia. Consultado en 30 de noviembre de 2012.

MAGRO FO; SALATA AC; BERTOLINI EV; CARDOSO AII. 2011. Produção de repolho em função da idade das mudas.RevistaAgro@ mbiente 5: 119-123.

MODOLON TA; BOFF P; BOFF MIC; MIQUELLUTI DJ. 2012. Homeopathic and high dilution preparations for pest management to tomato crop under organic production system. Horticultura Brasileira 30: 51-57.

ROSSI F; MELO PCT; AMBROSANO EJ; GUIRADO N; SCHAMMASS E. 2006. Aplicação do medicamento homeopático Carbo vegetabilis e desenvolvimento das mudas de alface. Cultura Homeopática 17: 14-17.

SARANDÓN S. 2008. La Agroecología: enfoque científico para un desarrollo rural sustentable. Disponible en http://www.agroecologia.inf. br/conteudo.php?vidcont. Consultado en 24 de junio de 2011.

SILVA JÚNIOR AA; MIURA L; YOKOYAMA S. 1988. Repolho: novas cultivares de verão. Agropecuária Catarinense 1: 47-49.

SOUZA APO; ALCÂNTARA RLC. 2003. Alimentos orgânicos: estratégias para o desenvolvimento do mercado. In: NEVES MF; CASTRO LT. Marketing e estratégia em agronegócios e alimentos. São Paulo: Atlas. 332-347. 